Estudios sobre el Mensaje Periodístico ISSN-e: 1988-2696

http://dx.doi.org/10.5209/ESMP.55602

\title{
Las ONGD reflejadas en el temario. Estudio comparativo de su imagen entre la etapa previa y posterior al impacto de la crisis económica
}

\author{
Carmen Costa-Sánchez'; José Antonio López Rey²
}

Recibido: 28 de enero de 2016 / Aceptado: 22 de julio de 2016

Resumen. El artículo estudia la imagen de las ONGD en el temario de La Voz de Galicia a lo largo de diez meses, con el objetivo de comparar si la crisis económica ha supuesto alguna variación en la imagen de este perfil de organizaciones. Se ha analizado la producción periodística de cinco meses de 2006 y de 2010. La metodología empleada ha sido el análisis de contenido. Se ha utilizado la prueba de Chicuadrado para mostrar la relevancia estadística de la relación entre las variables. Los resultados indican que las ONGD han sido más visibles en la etapa posterior a 2008. Confirman que han variado las temáticas a las que aparecen vinculadas y la existencia de un star system de entidades, que además se modifica debido a la crisis.

Palabras clave: ONGD; temario; imagen; producción informativa; crisis económica.

\section{[en] NGDO press coverage. Comparative study of their image in the pre- and} post-impact of the economic crisis

\begin{abstract}
This article examines the image of NGDO in La Voz de Galicia agenda along ten months for purpose of comparing if economic crisis has meant any difference for their image. Press coverage along five months of 2006 and the same period of 2010 have been analysed. Content analysis has been the methodology. Data crosses have employed Chi-squared test to show the statistical importance of the relation between the variables. The results indicate that ONGD have been more visible after 2008 . The topics to which they appear linked have changed and we can confirm the existence of a star system that has been modified because of the crisis.
\end{abstract}

Keywords: NGDO; agenda; image; informative production; economic crisis.

Sumario. 1. Introducción. Las ONGD en España. Necesidades comunicativas. Estudio de su imagen; 1.1. Estudios previos sobre la imagen de las ONG en el temario. 2. Materiales y metodología. 3. Resultados; 3.1. Presencia; 3.2. Visibilidad; 3.3. Secciones informativas; 3.4. Géneros periodísticos; 3.5. Autoría; 3.6. Temática; 3.7. Ranking de entidades; 3.8. Presencia en titular; 3.9. Fuente principal/ secundaria de la información; 3.10. Otras fuentes; 3.11. Tratamiento recibido. 4. Discusión y conclusiones. 5. Referencias bibliográficas.

Cómo citar: Costa-Sánchez, Carmen y López Rey, José Antonio (2017): “Las ONGD reflejadas en el temario. Estudio comparativo de su imagen entre la etapa previa y posterior al impacto de la crisis económica", en Estudios sobre el Mensaje Periodístico 23 (1), 375-390.

\footnotetext{
1 Universidade da Coruña.

E-mail: carmen.costa@udc.es

2 Universidade da Coruña.

E-mail: jlopezr@udc.es
} 


\section{Introducción. Las ONGD en España. Necesidades comunicativas. Estudio de su imagen}

Según Lasso de la Vega, la trayectoria de las ONGD españolas es parecida a la de otras organizaciones similares de Europa, teniendo en cuenta el retraso causado por nuestro menor grado de desarrollo y las dificultades políticas que durante la dictadura dificultaron su creación (Lasso de la Vega, 1993: 599). En este sentido, y desde una perspectiva cultural-histórica, tan importante como la dictadura franquista, y favorecida por ella, es el papel que la Iglesia Católica ha tenido en la historia de las ONGD y que es hegemónico (Orella, 2000: 61; Vinyes, 1997).

Con el fin de la dictadura dio comienzo un proceso de secularización que favoreció la pérdida de parte de esta hegemonía. A partir de la transición se produce un proceso de pérdida de poder y presencia de lo religioso en la vida social, favorecido por la aparición de otras fuerzas que empujaron para ocupar roles sociales más influyentes. En un primer momento fueron las organizaciones vinculadas a los partidos y sindicatos las que fueron creando ONGD que se sumaban a las de origen eclesial, pero después las profesionales (de educación, de salud) y, sobre todo, las solidarias (como las "sin fronteras") se ocuparon de laicizar la solidaridad internacional al partir de presupuestos éticos y no confesionales. La normalización de la situación política y la vertebración de la sociedad civil, así como el proceso de apertura al exterior, permitió que otros tipos organizacionales como las ONGD universitarias e internacionales tuvieran también su espacio en el sector.

El conjunto de ONGD en la actualidad se puede caracterizar a partir de unos rasgos concretos (López Rey, 2006a). Si nos ceñimos a las entidades que conforman la Coordinadora de ONGD de España se puede afirmar que es un sector joven, pues casi el $80 \%$ tiene menos de 30 años de antigüedad. Posee un origen social religioso dominante, con un tercio del total de las organizaciones creadas bajo esta inspiración. La forma asociativa (mayoritaria en los comienzos) ha ido perdiendo terreno frente al tipo jurídico de fundación. Mayoritariamente ubican su oficina central en Madrid, aunque también en Barcelona y en menor medida en Andalucía, Aragón, Castilla León, Extremadura, Navarra, País Vasco y Valencia. Una última consideración. Según Lasso de la Vega (1993: 603) la mayor parte de las organizaciones participa en múltiples y diversificados sectores. La expansión que muchas de ellas sufrieron - todas salvo las que mantuvieron su especialización inicial en temas de refugiados, derechos humanos, etc.- - las obligó a cubrir áreas no previstas inicialmente, lo que devino al final en una cierta uniformización. Dicho de otro modo, todas hacen un trabajo parecido, si bien la manera de hacerlo puede diferir de unas a otras. Cuestiones como el tamaño, el nivel de profesionalización, su complejidad interna, la cantidad de recursos (materiales y humanos) de que disponen introducen también matices importantes que sirven para diferenciar unas ONGD de otras.

La naturaleza y finalidad de las ONGD las conduce a la imprescindible necesidad de relacionarse, para lo que precisan incorporar la función de comunicación.

En lo que respecta a la relación con sus colaboradores, la comunicación interna contribuye a sumar fuerzas para el proyecto común, difundir la información que les afecta, promover la unión que da sentido a un trabajo de equipo. Dicha función resulta clave para una organización que trabaja desde el ejemplo para el conjunto social, con el objetivo de sensibilizar, por lo que sus integrantes se convierten en los principales embajadores de la filosofía de actuación y de los valores compartidos. Según 
Gómez Miliani (2006), el personal voluntario que estas organizaciones necesitan en el desarrollo de su labor requiere de un esfuerzo y una atención superior al personal contratado, pues la recompensa está en la motivación.

En cuanto a la comunicación externa, las relaciones con otros actores sociales se convierte en prioritaria, tanto para su supervivencia, es decir, para la obtención de recursos, como para lograr el reconocimiento, la colaboración o la concienciación social, es decir, la concienciación para el cambio social (Nos et al., 2012). Por ello buscan también la visibilidad en los medios (Castillo Esparcia, 2007).

El beneficio más instrumental de dicha visibilidad mediática se ha concretado en cifras. "Las entidades con DCYP (Departamentos de Comunicación y Prensa) obtienen 830.000 de diferencia media con respecto a las que no poseen dicho recurso organizativo. Los DCYP, pues, son muy útiles para las organizaciones que pretenden ocupar posiciones en el mercado material" (López Rey, 2006b: 46).

Pero más allá de eso, la comunicación se ha convertido en una herramienta estratégica para este conjunto de instituciones (Soria Ibáñez, 2011; Balas Lara, 2011; Padilla, 2009; González Luis, 2006a). "Es, sin duda, una herramienta estratégica que actúa en la búsqueda de objetivos concretos y que, por lo tanto, debe ser planificada y desarrollada por un conjunto de profesionales abocados a este fin" (Arroyo et al., 2010).

Para Etkin (2012), las entidades sin fines de lucro tienen un proyecto institucional que se sostiene interna y externamente a partir de ciertas estrategias de comunicación planificadas o no. Por ello han incorporado los correspondientes departamentos de comunicación en sus estructuras organizativas. En el estudio de Almaraz et al. (2010), en el que se radiografiaban los departamentos de comunicación de ONGD en España y Argentina, se detecta que aproximadamente un $80 \%$ de ambas muestras de ONG sí cuentan con un área especializada en desarrollar la comunicación de la organización.

Sin embargo, Balas Lara (2008) detecta que la gestión de la comunicación en las organizaciones del tercer sector no lucrativo, no resulta el punto fuerte de su actividad, dado que carecen de una estrategia de comunicación que transmita de manera coherente la misión de la organización a sus diferentes públicos (Balas Lara, 2008: 28). "La falta de comprensión de las diferencias y la adaptación a un modelo ya efímero de comunicación y sin validez para las organizaciones del Tercer Sector provocó que, a pesar de ser entidades con alta credibilidad, éstas tengan hoy en día el menor porcentaje de presencia y eficiencia en los medios" explica García Orosa (2006).

\subsection{Estudios previos sobre la imagen de las ONG en el temario.}

En el repaso que hace Soria Ibáñez (2011) al papel de las ONGD como fuente de información, la autora distingue tres etapas:

Una primera etapa — situada a finales de los 80 y principios de los 90 — en la que las informaciones procedentes de las ONG tenían que ver con las características de ellas mismas, con el fin de darse a conocer como organización.

Una segunda etapa, en la década de los 90, donde las informaciones estaban relacionadas con proyectos que llevaban a cabo en los países en vías de desarrollo. Esto se radicalizó hasta el punto de facilitar a los medios informaciones sobre situaciones 
extremas, acompañadas de documentos catastrofistas y desagradables, como la mejor forma de concienciación (González Luis, 2006b: 414-415).

Un tercer momento, en la actualidad, donde el papel de las oenegés es mucho más profesional y la información adquiere matices de concienciación y sensibilización (Soria Ibáñez, 2011: 159).

No obstante, las ONGD han estado presentes escasa y recientemente en la agenda investigadora por cuanto a sus relaciones con los medios e imagen en el temario. Se resumen a continuación los hallazgos más relevantes.

En el estudio de González Luis (2006b), respecto a la cantidad y frecuencia de noticias sobre ONGD en la prensa, se detecta una tendencia creciente en el conjunto del período analizado (1998-2003), teniendo lugar el incremento más significativo a partir del año 2000, lo que coincide con la creación de los departamentos de comunicación y la profesionalización de los mismos.

En el estudio de Lewis et al. (2006), se detecta que los actores gubernamentales y económicos son responsables de casi el $60 \%$ de los comunicados de prensa localizados en la cobertura informativa de la prensa británica, en contraposición a las $\mathrm{ONG}$, que solo alcanzan el $11 \%$.

En la investigación desarrollada por González Luis (2013), que pretende analizar el tratamiento otorgado por la prensa regional navarra a las informaciones relacionadas con la solidaridad, la cooperación y la acción de las ONGD, se llegan a una serie de resultados sobre imagen en el temario:

- La mayor parte de noticias se publican en Sociedad (40,6\%), seguida de Local $(21,7 \%)$ y Política $(12,3 \%)$.

- La noticia es el género prioritario a la hora de tratar estas cuestiones $(76,1 \%)$. La entrevista $(7,2 \%)$ y el reportaje $(6,6 \%)$ son minoritarios.

- Entre los dos periódicos publican una media de algo más de una noticia diaria sobre estos ítems. De ellas, más del $60 \%$ son pequeñas.

- La temática principal es el voluntariado, con un 12,5\%. La segunda temática más presente es el 'caso Urcheguía' (un supuesto delito de adquisiciones ilegales de terrenos por parte de una ONG con sede en el País Vasco), asunto que acapara el 9\% del total y evidencia la repercusión mediática que tienen las crisis de comunicación. Los proyectos desarrollados en terreno, con un 8,8\%, son la tercera temática con mayor presencia.

Entre sus conclusiones, apunta que las ONGD no ostentan el monopolio mediático en los ítems analizados, aunque son la fuente informativa más empleada en este tipo de coberturas (González Luis, 2013).

En el estudio de Lámy (2013), sobre el diario portugués Publico, se concluye que las oenegés no alimentan la actualidad informativa de forma regular, sino que aparecen en momentos y temas específicos, como los relacionados con derechos humanos o medio ambiente, lo que las excluye de la agenda mediática dominante.

El estudio de Murciano et al. (2010), centrado en el seguimiento de los temas de cooperación internacional en la prensa europea de referencia, confirma que dichas temáticas reciben en general un espacio reducido dentro de sus contenidos $(3,5 \%)$. Se apunta a que la cooperación tiene garantizado un espacio, limitado pero regular. El protagonismo de las $\mathrm{ONG}$ en el análisis realizado se ubica en cuarta posición, 
por detrás de gobiernos y agencias públicas nacionales, organismos multilaterales y actores de la cooperación descentralizada.

Powers (2014) considera que las oenegés desarrollan estrategias de relaciones con los medios distintas en función de cuatro elementos: obtención de fondos, relaciones con el estado, dinámicas organizacionales e impactos y audiencias deseadas. Detecta que existe una lucha entre estas entidades por la cobertura informativa, que no solo les resulta complicada de lograr, sino que crea desigualdades entre ellas, generando un star system de entidades (Powers, 2015).

\section{Materiales y Metodología}

El objetivo de la investigación que se presenta es el de conocer si se han producido variaciones en la presencia y visibilidad de las ONGD en el período anterior y posterior a la crisis económica de 2008. Es, por tanto, un objetivo de tipo comparativo, lo que requerirá la medición entre una etapa previa de bonanza económica (2006) y otra posterior a dicho año de referencia, considerado como tal por ser el de la caída del banco de inversiones norteamericano Lehman Brothers.

Se ha escogido el diario autonómico La Voz de Galicia (LVG) por ser cabecera autonómica de referencia (según OJD y EGM) y uno de los periódicos estatales más relevantes en el panorama informativo español. Asimismo por la necesidad de incluir en el análisis la sección de Local, de presencia habitual (González Luis, 2013) y necesaria (CONGDE, 2007) para este tipo de organizaciones. En este sentido, el periódico elegido presenta una marcada estructura geográfica que aplica en su tratamiento informativo diario ya que cuenta con trece suplementos locales, correspondientes a sus trece delegaciones territoriales de las que dispone a nivel Galicia. De todas las posibles, se eligió la edición local de A Coruña como la analizable, puesto que el estudio de todas ellas alteraría el modelo de periódico que llega a manos de un/a ciudadano/a medio, quien no accede diariamente a las trece ediciones locales, sino a la de su ámbito de referencia. Por otra parte, es en dicha provincia, en la que se localizan la mayoría de ONGD en Galicia (Costa-Sánchez, 2013).

En cuanto a la selección temporal, se escoge el año 2006 por ser un año de bonanza económica. Se elige 2010 para evitar la coincidencia con elecciones a nivel España (celebradas en 2011) y autonómicas (al año siguiente, 2012), además de por ser un año en el que ya habían comenzado a aplicarse los recortes.

El presente trabajo plantea la doble hipótesis de que, tras la crisis de 2008, las ONGD han visto modificada su presencia en el temario: por una parte, a nivel cuantitativo, es decir, ganando presencia en las páginas (h.1.1); por la otra, de forma más cualitativa, asociadas a lo local y a la colaboración con el entorno más próximo, en mayor medida que a otras temáticas, como los proyectos internacionales (h.1.2). Dicha hipótesis surge de la presuposición de que la crisis económica ha arrastrado a las oenegés al primer plano de la actualidad vinculadas al valor noticia "crisis", lo que las situaría en primera línea del escenario mediático.

La metodología aplicada ha sido el análisis de contenido. Se realizó por medio de una herramienta diseñada ad hoc para conocer cuantitativa y cualitativamente cómo aparecen reflejadas las ONGD en las páginas del diario analizado. Investigaciones previas (González Luis, 2006b y 2013) han contribuido al diseño de dicha herramienta, que se estructuró en dos grandes bloques: items formales, que singularizan el texto 
por las cuestiones de forma que se registran en diversos campos e items de contenido, que profundizan en el asunto tratado en la pieza periodística analizada. Dentro de los items formales se codificaban: fecha, día de la semana, mes, sección, autoría, género periodístico, módulos, página, ubicación en página, titular, tipo de titular y presencia de antetítulo, subtítulo, intertítulos, lead, fotografía y/o infografía. Dentro de los items de contenido se incluyeron: nombre de la ONGD, ámbito de actuación, tipo de fuente, otras fuentes de información, temática y tratamiento recibido.

El período de estudio abarcó un total de diez meses, en concreto: febrero, abril, junio, septiembre y noviembre de 2006 y de 2010. Se evitaron los meses de verano por ser meses de sequía informativa, lo que podía distorsionar los resultados. Investigaciones previas sobre imagen en el temario de distintos actores y fenómenos sociales han empleado períodos de estudio inferiores (Cheng et al., 2009; Igartua Perosanz et al., 2006; Román Portas, 2009) o muy similares (Arroyo Almaraz et al., 2013; González Luis, 2013).

En total, se revisaron 296 periódicos para identificar toda unidad periodística, entendiendo como tal cualquier texto no publicitario publicado en las páginas del diario, donde apareciese alguna oenegé, tanto en el grupo de titulación como en el cuerpo de la pieza y tanto en textos informativos como opinativos.

Para garantizar la replicabilidad que debe caracterizar a cualquier investigación, un $10 \%$ de la muestra de estudio fue codificada por un segundo investigador, lo que dio lugar a un índice de coincidencia del 94\%.

El programa utilizado en la sistematización de los datos fue el Adobe FileMaker Pro Advanced 14.0.1. Los cruces de datos que permiten extraer la representatividad estadística de los resultados se realizaron en SPSS.

\section{Resultados}

\subsection{Presencia}

El dato bruto de presencia indica que al menos se ha publicado un texto al día referente a oenegés en las páginas de $L V G$ y en el conjunto de sus secciones. Exactamente, una media de 1,08 textos/ día. Se trata de un cómputo global de 321 unidades periodísticas publicadas, en espacio ocupado poco más de 5.000 módulos, que, trasladados a páginas, equivaldrían a un total de 101,72 páginas relacionadas con estas organizaciones. Por años, la balanza se inclina ligeramente hacia 2006, pues en este año se publican 169 unidades periodísticas, frente a las 152 de 2010. En cuanto al espacio publicado, es ligeramente superior el difundido en 2010, en concreto 108 módulos más, equivalente a algo más de dos páginas de producción periodística (2.597 módulos en 2010, frente a los 2.489 en 2006).

\subsection{Visibilidad}

El análisis de la presencia resultó insuficiente y muy igualado en la comparativa 2006/ 2010. Por ello se consideró de interés prestar atención a la visibilidad de las unidades periodísticas analizadas.

Para analizar la visibilidad, se tomaron en cuenta dos ítems formales: el tipo de página (par/ impar) y la ubicación dentro de página (toda la página, recuadro su- 
perior izquierda, recuadro superior derecha, media página superior, media página inferior, recuadro inferior izquierda, recuadro inferior derecha). Se construyó así el índice de visibilidad de la información 1, que arrojó resultados estadísticamente significativos en relación con la variable año de publicación.

Se recategorizó la variable en cuatro posibles opciones: visibilidad muy baja, baja, media o alta, en función de la puntuación obtenida (de uno a quince puntos). Los resultados muestran que los textos difundidos en 2006 presentan una visibilidad muy baja, baja o media, en contraposición a los de 2010, que destacan por una alta visibilidad.

Gráfico 1. Índice de visibilidad de la información 1 recategorizado (\%). Elaboración propia.

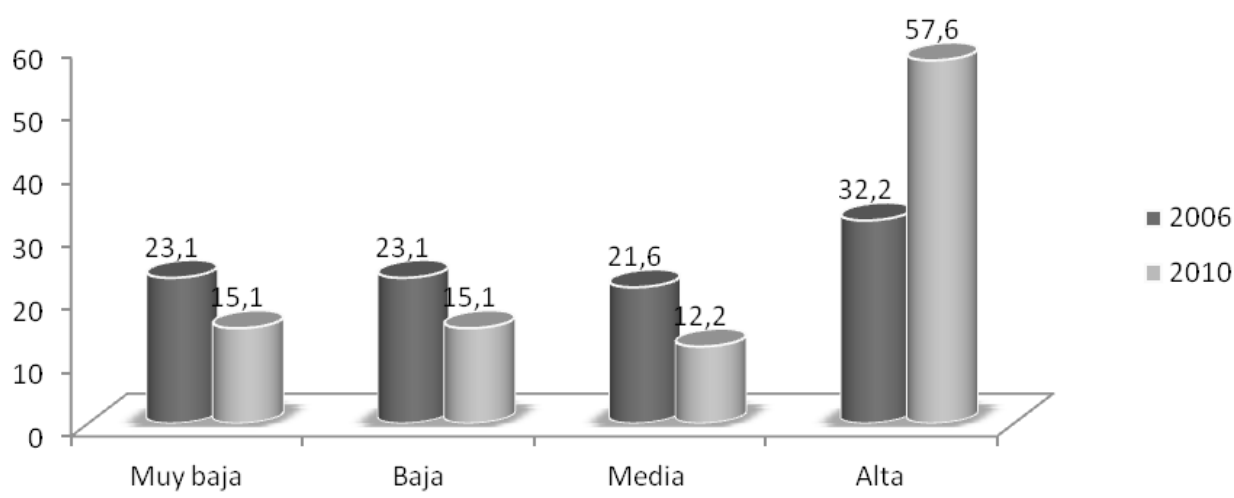

Se intentó ir un paso más allá con la construcción de un índice de visibilidad de la información 2, en el que se aplicaba la fórmula: $(\mathrm{A}+\mathrm{B}) \times \mathrm{C} \times \mathrm{D} \times \mathrm{E}$, de donde, $\mathrm{A}=$ Índice de relevancia de la información $1 ; \mathrm{B}=$ Elementos integrantes de la unidad (antetítulo, subtítulo, intertítulo, fotografía, infografía, lead); $\mathrm{C}=$ Número de módulos; $\mathrm{D}=$ Presencia Portada Local; E = Presencia Portada Exterior. La puntuación máxima que podía obtener cada unidad periodística eran 4.400 puntos.

Tabla 1. Cálculo del Índice de visibilidad de la información 2. Elaboración propia

\begin{tabular}{|c|l|c|}
\hline Id_en fórmula & \multicolumn{1}{|c|}{ Variable } & Valor máximo \\
\hline A & Índice de relevancia de la información 1 & 5 \\
\hline B & Elementos & 6 \\
\hline C & Número de módulos & 100 \\
\hline D & Portada Local & 2 \\
\hline E & Portada Exterior & 2 \\
\hline Total & Índice de visibilidad de la información 2 & 4400 \\
\hline
\end{tabular}

Este segundo índice de visibilidad de la información, sin embargo, no logra diferencias estadísticamente relevantes en relación con la variable año, probablemente porque los elementos que integran las unidades periodísticas son similares para 
ambos períodos y porque, aunque existe una ligera diferencia en módulos ventajosa para 2010, no es tan elevada como para que suponga diferencias reseñables en este sentido.

\subsection{Sección informativa}

Como si hubiese una asignación estable para las secciones temáticas disponibles, los datos indican una cuota semejante dedicada por año en cada una de las secciones informativas analizadas, destacando para ambos períodos la sección de Local como la de mayor presencia para este tipo de informaciones, seguida de Sociedad. La presencia de dichas organizaciones en Portada es residual para ambos períodos. El cruce año/ sección indica que no hay una relación estadísticamente representativa entre ambas variables (el nivel de significación fue de 0,9999).

Tabla 2. Sección informativa/ año (\%). Elaboración propia

\begin{tabular}{|c|c|c|c|c|}
\hline & \multicolumn{2}{|c|}{ Año } & \multirow{2}{*}{ Total } \\
\hline & & 2006 & 2010 & \\
\hline \multirow{11}{*}{ Sección } & España & $4,1 \%$ & $4,6 \%$ & $4,4 \%$ \\
\hline & Galicia & $11,8 \%$ & $12,5 \%$ & $12,1 \%$ \\
\hline & Internacional & $8,9 \%$ & $10,5 \%$ & $9,7 \%$ \\
\hline & Local & $36,7 \%$ & $34,2 \%$ & $35,5 \%$ \\
\hline & Opinión & $1,8 \%$ & $2,0 \%$ & $1,9 \%$ \\
\hline & Portada edición local & $1,2 \%$ & $1,3 \%$ & $1,2 \%$ \\
\hline & Portada exterior & $1,8 \%$ & $1,3 \%$ & $1,6 \%$ \\
\hline & Sociedad & $20,1 \%$ & $21,1 \%$ & $20,6 \%$ \\
\hline & Contraportada & $0,6 \%$ & $1,3 \%$ & $0,9 \%$ \\
\hline & Economía & $0,6 \%$ & $0,7 \%$ & $0,6 \%$ \\
\hline & Otra & $12,4 \%$ & $10,5 \%$ & $11,5 \%$ \\
\hline \multicolumn{2}{|r|}{ Total } & $100,0 \%$ & $100,0 \%$ & $100,0 \%$ \\
\hline
\end{tabular}

\subsection{Géneros periodísticos}

Predomina la noticia como el género al que más se han amoldado las informaciones relativas a oenegés. Sin embargo, en la comparativa entre ambos períodos, pueden encontrarse diferencias que se han mostrado como estadísticamente significativas. Destaca el hecho de que los géneros interpretativos - el reportaje y la crónica - son más habituales en 2010, mientras que el género informativo por excelencia - la noticia - tiene mayor presencia en la muestra de estudio correspondiente a 2006. En concreto, un 55\% de las unidades periodísticas en 2006 fueron noticias, frente al 37,5\% de 2010 (el descenso es considerable). Por el contrario, en 2010 hubo un $11,8 \%$ de crónicas (frente al $3 \%$ de 2006 ) y un $10,5 \%$ de reportajes frente al $5,9 \%$ de 2006. En artículos de opinión, entrevistas o breves, no se detectan diferencias remarcables entre ambos períodos. 


\subsection{Autoría}

Se había contemplado una triple posibilidad: a) que ésta fuese individual, es decir, que los nombres o iniciales de los autores de la unidad periodística compareciesen en el texto; b) que fuese colectiva, es decir, que la marca periodística La Voz (o Redacción) firmase la información sin dejar ver al autor o autora de la misma y c) que dicha información procediese de Agencias.

Los datos confirman de forma estadísticamente relevante que en 2010 existen más textos firmados con autoría individual, es decir, se ve potenciada la firma individual del periodista frente a 2006, cuando el protagonismo es para la firma colectiva generalmente englobada bajo las fórmulas La Voz y Redacción.

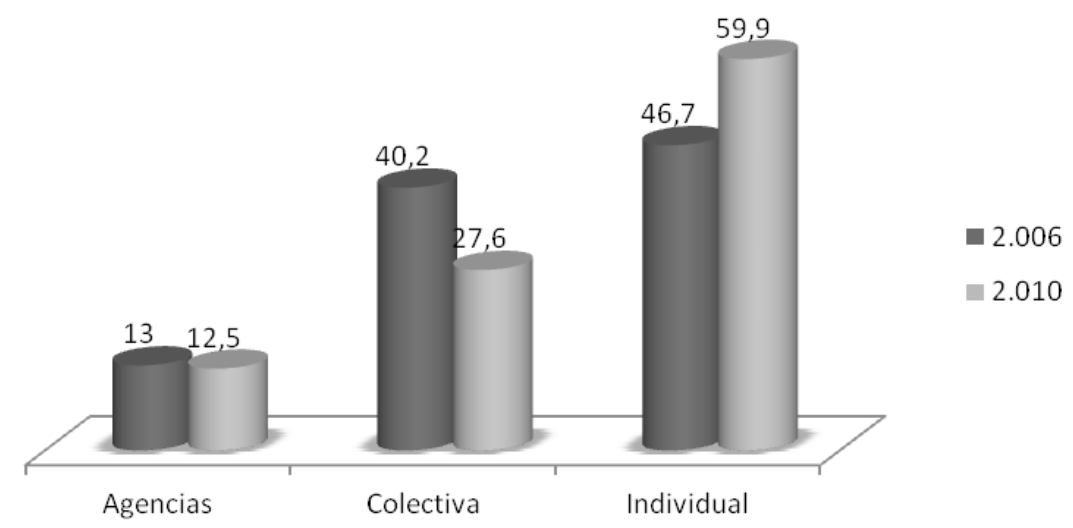

\subsection{Temáticas}

En la comparativa por años, puede observarse cómo las oenegés aparecen más vinculadas en 2006 a los temas de Inmigración, a los Proyectos de desarrollo en terreno y al Ecologismo, temáticas que ven significativamente menguada su presencia en el año 2010.

También los temas propios de la rutina de su actividad son cuantitativamente más relevantes en 2006, donde tienen más presencia las actividades desarrolladas con motivo de efemérides (Día mundial de), el hecho de presentar informes o estudios o los cursos, jornadas, charlas, conferencias o seminarios (adscritos a la categoría de Formación), que convocan.

En 2010, crece la temática de Acciones de Emergencia ligada a la catástrofe de Haití, que tiene lugar en enero. Además, incrementan mucho su presencia $(+11,8 \%)$ la temática de Necesidades sociales de la comunidad local en la que intervienen, resultado del empeoramiento de las condiciones de vida de los ciudadanos, así como las actividades organizadas con el objetivo de la captación de fondos $(+4,2 \%)$. 
Tabla 3. Temática/ año (\%). Elaboración propia

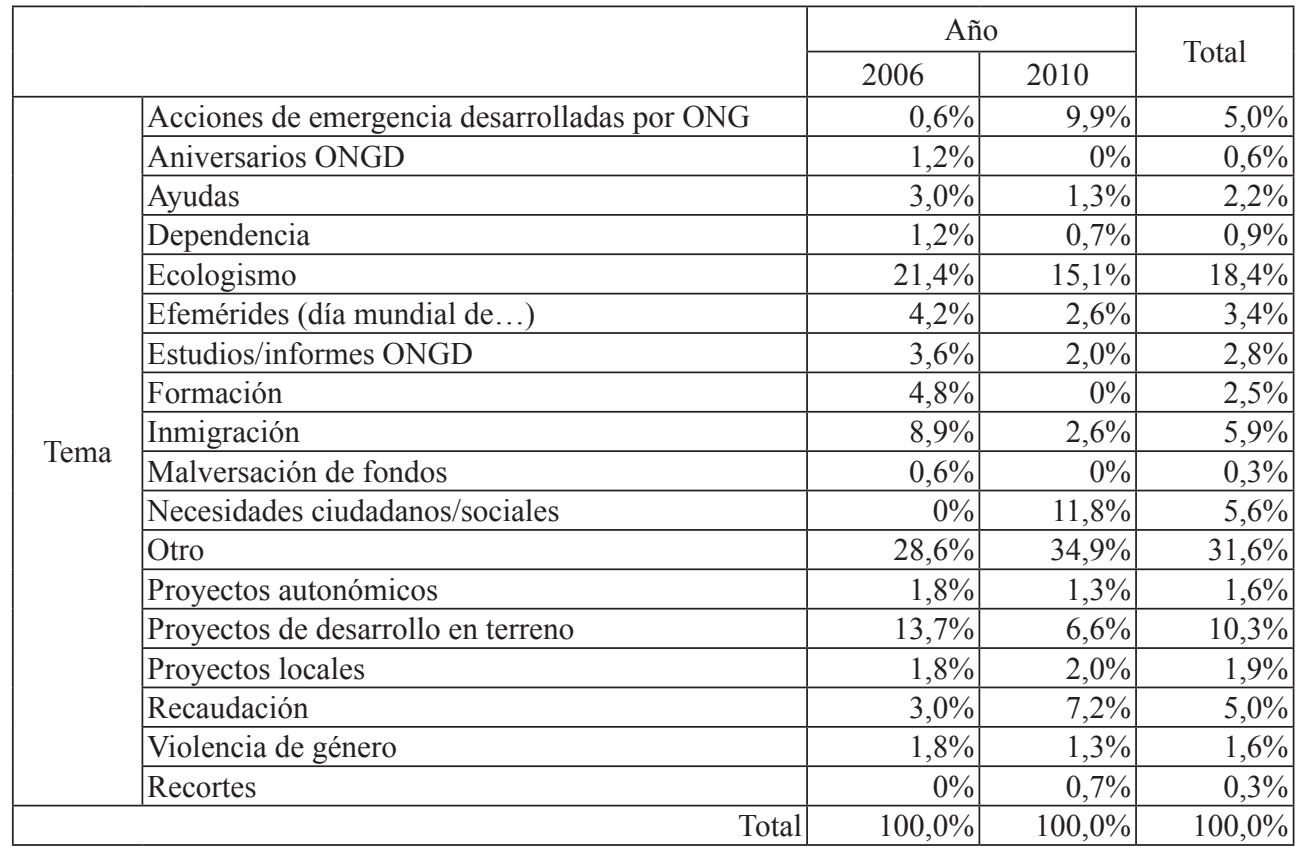

\subsection{Ranking de entidades}

Dado el elevado número y tipología de organizaciones detectadas, se han recategorizado todas las oenegés aparecidas en ocho grandes categorías que, en un segundo momento, se han puesto en relación con la variable año de referencia (2006/2010).

La recategorización ha dado lugar a estas ocho posibles adscripciones:

1. Varias, en el caso de que apareciese más de una entidad.

2. Genérico oenegé, para aquellas unidades periodísticas en las que no se especificase una denominación concreta, sino que hiciesen referencia al colectivo.

3. Cruz Roja, que se ha considerado a modo independiente, por el amplio protagonismo que se detectaba en el total estudiado.

4. Derechos Humanos/ Internacional, para aquellas entidades que trabajan a un nivel internacional en la defensa de los valores y derechos humanos para lograr un mundo más justo.

5. Local, en el caso de las oenegés que desarrollan su actividad en un ámbito local y en contacto con la comunidad más próxima.

6. Otra, sino era adscribible a ninguna de las categorías propuestas.

7. Ecologistas, para aquellas oenegés que desarrollan su labor principal en la lucha y en el cuidado del medio ambiente.

8. ONGD, para aquellas organizaciones que trabajan en proyectos de acción social.

Las diferencias indican que en el 2006 han tomado un mayor protagonismo las oenegés de tipo ecologista $(26,5 \%$ en 2006 y $17,7 \%$ en 2010$)$ y las de derechos humanos/ internacional (baja de 6,6\% a 4,1\% en 2010) mientras que, en 2010, han 
destacado las ONGD (33,3\% frente al 23,5\% de 2006), así como aquellas organizaciones que han desarrollado su labor en el entorno local (experimentan una subida del 10,3\%). La Cruz Roja se erige como categoría aparte, por ser denominador común para ambos períodos.

Gráfico 3. Tipo de ONG/ año (\%). Elaboración propia

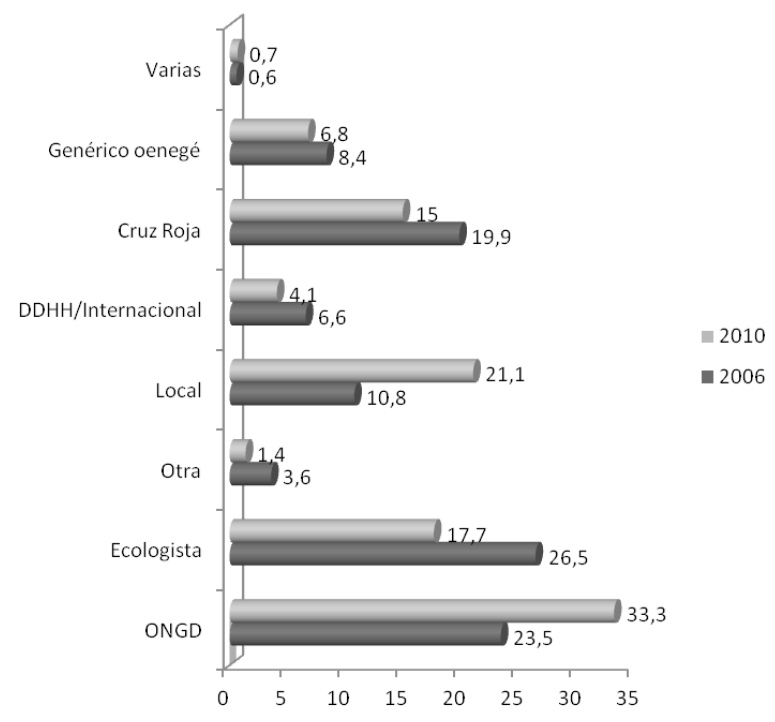

\subsection{Presencia en titular}

En la comparativa 2006/2010, los datos indican diferencias estadísticamente representativas. En 2006, acaparan más titulares que en 2010, en concreto en 2006 en un $36,1 \%$ de unidades periodísticas figuraba el nombre de la organización en titular frente a un $21,1 \%$ en $2010(-15 \%)$.

\subsection{Fuente principal/ secundaria de la información}

Los resultados indican que las oenegés han sido fuente de información principal en un $60 \%$ de la muestra de estudio en 2006 y en un 45,6\% en 2010. Han sido fuente secundaria en un $40 \%$ de los casos en 2006 , porcentaje que asciende al $54,4 \%$ para 2010. Por lo tanto, mientras en 2006 han sido fuente principal de la información, en 2010 han compartido voz en las unidades periodísticas con otros actores sociales.

\subsection{Otras fuentes}

Se ha mostrado como estadísticamente representativa la diferencia entre las fuentes a las que aparecen vinculadas en 2006 y en 2010. La lectura de dicha comparativa permite destacar el incremento tanto de los beneficiarios, como de los ciudadanos en 
el año 2010, categorías de fuentes que apenas se detectaban en 2006. Por otra parte, el incremento también es muy notable en el caso de las fuentes oficiales locales. Las fuentes estatales se mantienen y las fuentes oficiales autonómicas se reducen a la mitad con respecto a 2006.

Gráfico 4. Otras fuentes/ año (\%). Elaboración propia

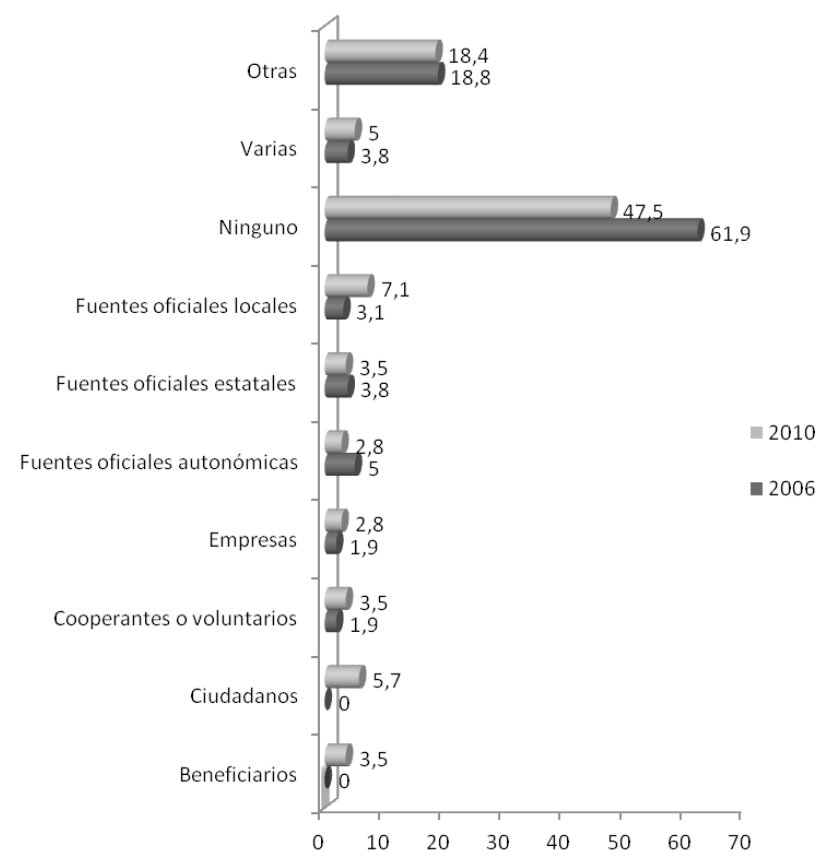

\subsection{Tratamiento recibido}

La mayoría de las unidades periodísticas analizadas aplican un tratamiento neutral a la cobertura informativa de las oenegés. No obstante, se muestran las diferencias significativas entre 2006 y 2010, siendo superior el tratamiento en positivo para el primer período estudiado $(12,6 \%$ de unidades periodísticas con tratamiento positivo en 2006 , frente al $6,1 \%$ de 2010 ).

\section{Discusión y conclusiones}

Además de una presencia global reducida y dispersa, puede concluirse que las oenegés han estado más presentes en número de unidades periodísticas en 2006, aunque menos en cuanto al espacio ocupado respecto a 2010. Más impactos, menos espacio. La hipótesis inicial, por lo tanto, que sostenía que las oenegés adquieren mayor presencia cuantitativa en el temario de $L V G$ en el período posterior a 2008, no se 
confirma, se apunta a un estancamiento o cuota de presencia semejante para ambos períodos.

Hay que destacar que se ha revelado como de utilidad la utilización de forma complementaria del concepto de visibilidad. Se puede estar muy presente y poco visible, el matiz, en términos de imagen, resulta de interés. Se considera un acierto metodológico haber incorporado este ítem de análisis, en función de las categorías que han permitido construir el índice de visibilidad 1. La investigación permite confirmar que las oenegés tienen mayor visiblidad en el período correspondiente a 2010 que respecto a 2006.

En segundo lugar, respecto de la segunda subhipótesis planteada, que establecía que las oenegés comparecen en el período post-crisis de forma diferente, más vinculadas a temáticas ciudadanas locales que internacionales, dicha hipótesis se confirma. La agenda temática ha variado. En la comparativa por años, se observa que los temas principales en 2006 son el Ecologismo, la Inmigración y los Proyectos de desarrollo en terreno. Los temas que podemos calificar como "de rutina" están también más presentes en 2006. En 2010, crecen especialmente las necesidades sociales locales.

Puede hablarse, por tanto, de una sustitución en la agenda del medio de los temas noticiosos vinculados a estas organizaciones, en paralelo al cambio producido en la realidad conocida por el mismo, de forma que en 2010 pierden peso las anteriores temáticas y se incrementan las acciones de emergencia (por la catástrofe de Haití), las necesidades socioeconómicas de la comunidad y las actividades para la recaudación de fondos.

Uno de los hallazgos de la presente investigación, que no formaba parte de la hipótesis principal, es que existen una serie de indicadores que conducen a concluir que las oenegés han liderado el proceso informativo en mayor medida en 2006 que en 2010. Este conjunto de indicadores está conformado por:

a) El predominio del género informativo.

b) El predominio de una autoría anonimizada bajo fórmulas colectivas.

c) El hecho de que sean fuente de información única y principal en mayor medida en 2006.

d) El hecho de que reciban un tratamiento en positivo mayor y hayan acaparado más titulares en 2006.

Ello conduce a una doble explicación. Por una parte, la desaparición o pérdida de recursos como resultado de las dificultades económicas. Ello habría afectado a sus estructuras de comunicación, de manera que en 2006 éstas se encontraban profesionalizadas y en su mejor momento, pero en 2010, resultado de la reducción de los recursos, los departamentos de comunicación desaparecen o pierden peso en los organigramas de dichas organizaciones con lo que la cobertura periodística de su actividad se vería afectada y pasaría a ser menos proactiva. Debe tenerse en cuenta, por ejemplo, que las notas o conferencias de prensa suelen transformarse en género informativo y raramente en género interpretativo. También explica el tratamiento informativo en positivo y el mayor protagonismo recibido en el período de estudio de 2006.

Por otra parte, el impacto de la crisis económica en el entorno social coloca este tema en primera línea de la agenda de la producción informativa, por lo que el medio asume un rol más proactivo en la inclusión en el temario de dicha temática (es más 
sensible a esta cuestión) y en el tratamiento de este tipo de informaciones. Por eso también el periodista cubre y contrasta más fuentes de información, perdiendo desde la oenegé el control informativo y generando en consecuencia textos más interpretativos.

Por supuesto, una futura línea de investigación queda planteada en este punto. Se trata de determinar si las estructuras de comunicación de las oenegés se han visto afectadas por la crisis económica, es decir, si desaparecen, si menguan sus recursos, si otros departamentos de la organización asumen su labor.

Todo lo anterior, paradójicamente, conduce a una conclusión hasta cierto punto positiva pues, a pesar de que se refleja que han perdido el liderazgo del proceso informativo, logran niveles similares en presencia y superiores en visibilidad a la etapa previa a 2008. Eso sí, perdiendo o cediendo el control del mensaje y con un cambio en los temas y roles que venían desempeñando.

Esto se explica por la irrupción de un tema en agenda, la crisis económica, que antes no existía y que tiene un efecto arrastre de forma que los medios acuden a cubrir escenarios y situaciones en las que, de una u otra forma, está presente este perfil de entidades. Y otras.

Por eso la investigación detecta que comparten discurso con otras voces en el período posterior a 2008. Es consecuencia directa de esa cesión en el mensaje. Destaca el protagonismo que asumen las fuentes oficiales locales, los ciudadanos, los beneficiarios o los voluntarios de dichas organizaciones.

Además, la investigación permite concluir la existencia de un star-system de entidades que varía para el período pre- y post- crisis económica.

Primeramente, cabe matizar que la atomización existente en el sector tiene su reflejo en el temario, pues hay múltiples organizaciones que aparecen en una única unidad periodística, pero no son fuentes habituales del ámbito informativo. Se encuentran en la periferia informativa. Esto se produce en ambos períodos de estudio y confirma la competencia dentro del sector por lograr una mayor presencia en el mercado informativo (López Rey, 2006a), con la intención de que ello repercuta en una mejora de la situación de la entidad en cuanto a todo tipo de recursos (humanos, económicos, etc.) y en su labor de concientización social.

Puede observarse que aquellas organizaciones con marcas más conocidas están también más presentes, lo que consolida la tendencia hacia un binomio indisoluble entre organización conocida y organización legitimada por el medio. Representan éstas el conjunto de organizaciones que logran salirse de la periferia informativa y se convierten en fuentes habituales y, en esa medida, se ve reforzada su presencia. Podríamos hablar de una jerarquía de fuentes dentro del ámbito de la cooperación, el voluntariado y la acción social al igual que en otras áreas informativas. La diferenciación en los rankings está relacionada con la diferenciación en las temáticas prevalentes para uno u otro período.

En 2006, predominan las oenegés vinculadas a los temas de ecologismo (Greenpeace o Ecologistas en acción), Inmigración y proyectos de desarrollo en terreno (Tierra de Hombres, Solidaridad Internacional, Ecodesarrollo Gaia). En 2010, destaca el protagonismo de organizaciones vinculadas a las necesidades socioeconómicas de los ciudadanos, especialmente de Cáritas, que se convierte en la oenegé de referencia (en menor medida Unicef) y aquellas vinculadas a lo acontecido en Haití (Médicos sin fronteras). La marca oenegé prevalente para 2006 es Greenpeace, Caritas para 2010 . 


\section{Referencias bibliográficas}

Arroyo, Isidoro; Baladrón, Antonio J.; y Martín, Rebeca (2013): “La comunicación en redes sociales: percepciones y usos de las Ong españolas". Cuadernos.info, 32, 77-88. http:// dx.doi.org/10.7764/cdi.32.497

Arroyo, Isidoro; Martín, Rebeca; y Mamic, Liliana (2010): “Los Departamentos de Comunicación de las ONG. Investigación comparativa entre Argentina y España”. Telos, 85, 130-143.

Balas, Monserrat (2011): La gestión de la comunicación en el Tercer Sector. Madrid, Esic Editorial.

CONGDE [Coordinadora de ONG para el Desarrollo-España] (2007): Los medios de comunicación y las ONGD: situación actual y retos. Madrid, CONGDE. http://www. mediosysida.org/download/publ1.pdf [Consulta: 10 de abril de 2015]

Castillo, Antonio (2007): "Relaciones Públicas en las organizaciones no gubernamentales". Sphera Pública, 7, 193-210.

Cheng, Lifen; Igartua Perosanz, Juan José; Palacios, Elena; Acosta, Tania; Otero, José Antonio; et al. (2009): “Aversión vs. Aceptación, dos caras de la misma moneda: un estudio empírico de encuadres noticiosos sobre inmigración en la prensa regional de Castilla y León". Zer: Revista de estudios de comunicación, 26, 35-57.

Costa-Sánchez, Carmen (2013): “ONGDs y prensa: estrategias de territorialización de la información en Galicia”. Sphera Publica, 1 (13), 136-165.

Etkin, Eugenia (2012): Comunicación para organizaciones sociales: de la planificación a la acción. Buenos Aires, La Crujía.

García Orosa, Berta (2006): “Aproximación teórica a la comunicación en el Tercer Sector. La necesaria reclasificación de la comunicación organizacional". Telos: Cuadernos de comunicación e Innovación, 69, 51-59.

Gómez Miliani, Cecilia (2006): El marketing como herramienta para la gestión en las organizaciones no gubernamentales ambientales de Venezuela. Tesis Doctoral. Málaga, Universidad de Málaga. http://goo.gl/Pg2LvJ [Consulta: el 14 de junio de 2015]

González-Luis, Hildegart (2006a): Estrategias de comunicación en las ONG de desarrollo: departamentos, funciones e impacto en los medios. Madrid, Cideal.

González-Luis, Hildegart (2006b): “La comunicación: herramienta estratégica en la misión de las ONGDS”. FISEC-Estrategias, Año II, 5, 31-53.

González-Luis, Hildegart (2013): "Radiografía de la presencia de la solidaridad, la cooperación, el desarrollo, y las ONGD en la prensa regional navarra". Estudios sobre el Mensaje Periodístico, 19 (1), 147164. http://dx.doi.org/10.5209/rev_ESMP.2013.v19.n1.42512

Igartua Perosanz, Juan José; Otero Parra, José Antonio; y Muñiz Muriel, Carlos (2006): "El tratamiento informativo de la inmigración en la prensa y la televisión española. Una aproximación empírica desde la Teoría del Framing”. Global Media Journal México, 3 (5), 1-15.

Lamy, Sonia (2013): “As ONGs na imprensa. As fontes não governamentais na imprensa diária”. Sur le journalisme About journalism Sobre jornalismo, 2 (1), 42-51. http://goo. gl/qejYJ8 [Consulta: 2 de mayo de 2015]

Lasso de la Vega, Alfonso (1993): "Las organizaciones no gubernamentales de desarrollo (CONGD): Un compromiso con el Sur”. In Rodríguez Piñeiro. M. et.al.: El sector no lucrativo en España (pp. 583-623). Madrid, Escuela Libre Editorial.

Lewis, Justin; Wilson, Andrew; Franklin, Robert Arthur; et al. (2006): "The Quality and Independence of British Journalism: Tracking the Changes Over 20 Years". Mediawise, 1. (http://goo.gl/CedMSX) [Consulta: 10 de junio de 2015] 
López Rey, José Antonio (2006a): El tercer sector y el mercado: conflictos institucionales en España. Madrid, Centro de Investigaciones Sociológicas (CIS).

López Rey, José Antonio (2006b): "Los medios de comunicación y ONGs: su importancia en la conformación de una nueva cultura corporativa en el sector solidario". Documentación Social, 140, 39-55.

Murciano, Marcial (dir. 2010): La prensa y la cooperación internacional. Cobertura de la cooperación para el desarrollo en nueve diarios europeos. Sevilla, Comunicación Social.

Nos, Elisa; Iranzo, Amador; y Farné, Alessandra (2012): "La eficacia cultural de la comunicación de las ONGD: los discursos de los movimientos sociales actuales como revisión". Cuadernos de Información y Comunicación, 17, 209-237.

Orella, José Luis (2000): “Desarrollo histórico”. En Ruiz Olabuénaga, J. I. (dir.): El sector no lucrativo en España (pp. 45-77). Bilbao, Fundación BBV.

Padilla Albaladejo, Adriana (2009): Las ONG y su función comunicativa: La escasa atención de los medios de comunicación a las temáticas solidarias. Madrid, Universidad CEUSan Pablo. http://goo.gl/Ydjyuj [Consulta: 14 de junio de 2015]

Román Portas, Mercedes (2009): “Tratamiento de derechos humanos en La Voz de Galicia”. Revista Latina de Comunicación Social, 64. http://dx.doi.org/10.4185/RLCS-64-2009864-819-826

Powers, Matthew (2015): "Contemporary NGO-Journalist Relations: Reviewing and Evaluating an Emergent Area of Research”. Sociology Compass, 9 (6), 427-437.

Powers, Matthew (2014): "The structural organization of NGO publicity work: Explaining divergent publicity strategies at humanitarian and human rights organizations". International journal of communication, 8, 90-107.

Soria Ibáñez, Mar (2011): La comunicación en las ongs españolas: la influencia de internet en el modelo estratégico de relaciones con los públicos. Málaga, Universidad de Málaga.

Vinyes, Ricard (1997): Aproximación histórica a las asociaciones de carácter no lucrativo en el ámbito de los servicios sociales. En Rodríguez Cabrero, G. y Codorniú, M. (dirs.): Las entidades voluntarias en España. Institucionalización, estructura económica y desarrollo asociativo (pp. 35-100). Madrid, Ministerio de Trabajo y Asuntos Sociales.

Carmen Costa-Sánchez es Profesora Ayudante Doctora de la Facultad de Ciencias de la Comunicación. Universidade da Coruña.

José Antonio López Rey es Profesor Titular de la Facultad de Sociología. Universidade da Coruña. 